\title{
Kinetic theory aspects of non-equilibrium liquid-vapor flows
}

\author{
Aldo FREZZOTTI* and Paolo BARBANTE** \\ * Department of Aerospace Science \& Technology, Politecnico di Milano \\ Via La Masa 34, 20156 Milano, Italy \\ E-mail: aldo.frezzotti@polimi.it \\ ** Department of Mathematics, Politecnico di Milano \\ Via Bonardi 9, 20133 Milano, Italy
}

\section{Received: 1 October 2016; Revised: 29 November 2016; Accepted: 3 January 2017}

\begin{abstract}
Kinetic theory of fluids plays an important role in understanding and modeling mass, momentum and energy transfer between the vapor and liquid phase in non-equilibrium two-phase flows, in which evaporation and/or condensation take place. The paper presents a review of the literature which focuses on kinetic modeling of the vapor-liquid interface. Starting from the studies of the Knudsen layer structure in evaporation and condensation, the problem of the formulation of kinetic boundary conditions is described and discussed. The formulation of models based on approximate kinetic descriptions of dense fluids is described and the model capabilities are assessed through the analysis of the results obtained by various authors.
\end{abstract}

Key words : Evaporation, Condensation, Kinetic theory, Boundary conditions

\section{Introduction}

The amount of research papers devoted to experimental, theoretical and numerical studies of two-phase flows and, in particular to evaporation and condensation phenomena, is huge. Hence, it is necessary to declare immediately the aims and contents of the present review, which only focuses on the kinetic theory aspects of modeling liquid-vapor flows out of equilibrium (Ytrehus, 1997; Sone, 2000). The decision of restricting the present review focus is made easier by recent publications (Persad and Ward, 2016), where the experimental research activity is properly reviewed.

The first aim of the present review is to briefly summarize the state of the art of kinetic models for evaporation and condensation flows as well as to try to insert the developed theory in the more general context of two-phase flows modeling which had a strong development in the last few years. As it will be described below, the impact of the consistent kinetic description on two-phase flow modeling has been quite limited so far. However, the assessment of the accuracy and sensitivity of kinetic models in describing mass, momentum and energy transfer through vapor-liquid interfaces cannot be completed until such models are inserted into two-phase flow simulation codes and used to investigate flow configurations definitely more realistic than those used to develop the models themselves.

The second aim is to extend the presentation of kinetic models to theories that allow a unified treatment of the vapor and liquid phase by a single kinetic equation, although adopting simplifying assumptions. Their original formulation and following theoretical analysis can be found in relatively old papers (De Sobrino, 1967; Grmela, 1971) and (Karkheck and Stell, 1981). More recently, similar ideas and formalism have been the starting point of investigations of liquid flows in nano-pores (Davis, 1992) as well as of computational models of two-phase flows, based on Lattice-Boltzmann schemes (He and Doolen, 2002) and Dissipative Particles Dynamics (Tiwari and Abraham, 2008). It is also possible to include the Consistent Boltzmann Algorithm (CBA), proposed in Alexander et al. (1997), in the same family of models capable of unified kinetic description of two-phase systems (Hadjiconstantinou et al., 2000). The interest in considering this class of models is due to the following reasons: (a) their numerical implementations, based on stochastic particles schemes (Alexander et al., 1997; Frezzotti et al., 2005), run usually faster than Molecular Dynamics (MD) simulations using the 
same particles number; (b) their estimation of evaporation coefficients (Frezzotti et al., 2005; Kon et al., 2014) and the properties of vapor-liquid interfacial scattering are very close to corresponding MD results for a simple fluid (Kobayashi et al., 2012); (c) they allow extending the applicability of kinetic theory concepts to non-ideal vapor phases, although approximately; (d) applications of moment method approximations allow obtaining semi-analytical solutions of simple flows (Davis, 1992) and, finally,(e) they provide a microscopic basis (Piechór, 2008) to derive Diffuse Interface Models (Anderson et al., 1998) of two-phase flows. According with the previous considerations, the present review is given the following structure: Section 2 is devoted to a concise summary of the results of studies of evaporation and condensation flows, in the context of ideal vapor flows, described by the Boltzmann or kinetic model equations (Cercignani, 1988); Section 3 describes how the theory developed in the studies described in Section 2 has been or could be applied to vaporliquid flows, in combination with other computational techniques; Section 4 is devoted to a description of basis of EnskogVlasov model equation (De Sobrino, 1966; Grmela, 1971; Karkheck and Stell, 1981) and CBA scheme (Alexander et al., 1997). Applications of Enskog-Vlasov model are described in Section 5, whereas concluding remarks and comments are given in the final Section 6.

\section{Kinetic theory applications to evaporation and condensation of dilute vapors}

Kinetic theory of fluids plays an important role in the formulation of mathematical/numerical models for mass, momentum and energy transfer between the vapor and liquid phases in non-equilibrium two-phase flows. In such situation, the velocity distribution function (Cercignani, 1988) of molecules spontaneously emitted from the liquid into the vapor phase differs, to some extent, from the velocity distribution function of molecules of the vapor phase going to impinge on the liquid surface. The resulting discontinuity causes the formation of a kinetic layer (Knudsen layer) (Cercignani, 1988), whose thickness strongly depends on the flow regime, extending up to a few hundreds mean free paths in the case of the strong evaporation caused by laser induced ablation of materials (Ohse et al., 1980). At the level of macroscopic scales, the presence of kinetic layers manifests in the form of sudden jumps suffered by macroscopic field variables (density, mean velocity, temperature...) appearing in hydrodynamic equations. Since the works of Hertz (1882) and Knudsen (1915), kinetic theory methods have been applied to investigate the Knudsen layer structure in evaporation and condensation flows, in order to correct the phenomenological nature of Hertz-Knudsen and Schrage formulas (Schrage, 1953). In particular, the steady, one-dimensional Boltzmann or kinetic model equations have been solved by a variety of analytical and numerical methods, either in the linearized or in the full non-linear form, to study evaporation or condensation in the half-space occupied by the vapor and obtain jump formulas, fully consistent with the assumed physical and mathematical model. It is to be noted that considering steady and one-dimensional solutions allows using the derived jump relationships also in unsteady flows and/or when the liquid-vapor interface is not planar. Actually, a quasi-steady and locally one-dimensional description holds when hydrodynamic regions evolve slowly on the time scale of the mean free time (Cercignani, 1988) and the Knudsen layer thickness is small when compared with the curvature of the liquid-vapor interface. The state of the art of the fundamentals of kinetic theory analysis of evaporation and condensation as well as of some of their applications are very well described in Ytrehus (1997) and Sone (2000).

Most of the fundamental results obtained or mentioned in the references quoted above have been derived under the assumption that the vapor phase consists of a simple dilute monatomic gas. Although many general flow properties do not depend on the number of internal molecular degrees of freedom or the number of distinct chemical species in the fluid, the flow details and intensity of jumps across kinetic layers are affected by the specific nature of component species. Extensions to polyatomic gases have been considered in Cercignani (1980), Soga (1985), Frezzotti and Ytrehus (2006) and Frezzotti (2007). More precisely, moment method solutions of a kinetic model equation have been obtained in Cercignani (1980) to study the strong evaporation of a polyatomic gas into a half-space. A more accurate solution method has been later applied in Soga (1985) to the linearized version of a similar kinetic model to obtain solutions asymptotically approaching a hydrodynamic state consistent with Navier-Stokes-Fourier equations. Linearized jump formulas have been obtained, in addition to macroscopic flow profiles. The non-linear Boltzmann equation, for a gas with rotational degrees of freedom, has been used to study steady evaporation and condensation in a half-space in Frezzotti (2007) and Frezzotti and Ytrehus (2006). The important case of the evaporation of multi-component substances has been considered in Frezzotti (1991), Sibold and Urbassek (1993) and Frezzotti (1997a) by numerical solutions of systems of Boltzmann equations. In particular, jump formulas for temperature, density and mixture composition are given in numerical form in Frezzotti (1997a) for half-space problem in which the fluid achieves a Maxwellian state at the end of the Knudsen layer. More general expressions to match kinetic layers with Navier-Stokes equations for a binary mixture are given in Takata and 
Golse (2007). It is difficult to overemphasize the importance of considering mixtures evaporation/condensation when the vapor flows in a volume occupied by a different and non-condensible species as, for instance, in Aoki et al. (1998) and Kosuge et al. (2010) for monatomic species. Extension to a binary mixture of polyatomic gases has been studied in Shishkova et al. (2013). It is interesting to note that the importance of this case also extends to modeling gas flow induced by gas absorption at walls of vacuum devices and systems (Frezzotti, et al., 2014), thanks to a transformation which turns absorption into condensation flows by properly relating the probability of molecular sticking at walls to an effective condensation coefficient.

\subsection{The problem of kinetic boundary conditions}

The studies mentioned above aimed at obtaining the Knudsen layer structure next to the vapor-liquid interface as well as the jump formulas and the correct conditions to match the hydrodynamic regions in the liquid and in the vapor across the kinetic layer. Hence, the detailed physical nature of the vapor-liquid interface was ignored and, accordingly, replaced with a simplified model, essentially based on the two following assumptions:

(1) - The vapor-liquid interface is a structureless geometrical surface, characterized by the local temperature $T_{I}$ and, possibly, by a local velocity $\boldsymbol{u}_{I}$ often set equal to zero, for simplicity.

(2) - The distribution function of molecules entering the vapor phase, at a location $\overline{\boldsymbol{r}}$ on the interface, is assumed to have the following form:

$$
(\boldsymbol{v} \cdot \boldsymbol{n}) f(\overline{\boldsymbol{r}}, \boldsymbol{v} \mid t)=(\boldsymbol{v} \cdot \boldsymbol{n}) f_{e}(\overline{\boldsymbol{r}}, \boldsymbol{v})+\int_{\left(\boldsymbol{v}_{1} \cdot \boldsymbol{n}\right)<0} R\left(\boldsymbol{v}, \boldsymbol{v}_{1}\right)\left|\boldsymbol{v}_{1} \cdot \boldsymbol{n}\right| f\left(\overline{\boldsymbol{r}}, \boldsymbol{v}_{1} \mid t\right) d^{3} \boldsymbol{v}_{1}, \quad \boldsymbol{v} \cdot \boldsymbol{n}>0
$$

Equation (1) assumes that the local flux emerging from a liquid surface element of normal $\boldsymbol{n}$ results from two distinct contributions. The first one consists of molecules belonging to liquid phase and spontaneously emitted into the vapor phase with distribution function $f_{e}(\bar{r}, \boldsymbol{v})$. The second one is due to the molecules of the vapor phase which are scattered back into the vapor region after a "short duration" interaction with the liquid phase. Such process is described by the scattering kernel $R\left(\boldsymbol{v}, \boldsymbol{v}_{1}\right)$ assigning the probability that molecules within the volume $d^{3} \boldsymbol{v}_{1}$, centered on $\boldsymbol{v}_{1}$, are scattered into the volume $d^{3} \boldsymbol{v}$, centered on $\boldsymbol{v}$. Because of the difficulties of giving Eq. (1) a more precise form on ground of first principles, it is usually postulated that $f_{e}(\boldsymbol{v})$ is the half range Maxwellian defined as follows (dropping $\overline{\boldsymbol{r}}$ ):

$$
f_{e}(\boldsymbol{v})=\sigma_{e} \frac{n_{I}}{\left(2 \pi R T_{I}\right)^{3 / 2}} \exp \left(-\frac{\boldsymbol{v}^{2}}{2 R T_{I}}\right), \quad \boldsymbol{v} \cdot \boldsymbol{n}>0,
$$

whereas Maxwell's gas-surface scattering kernel is the usual choice to describe molecular scattering from the condensed phase:

$$
R\left(\boldsymbol{v}, \boldsymbol{v}_{1}\right)=\left(1-\sigma_{c}\right)\left\{\alpha(\boldsymbol{v} \cdot \boldsymbol{n}) \frac{1}{2 \pi\left(R T_{I}\right)^{2}} \exp \left(-\frac{\boldsymbol{v}^{2}}{2 R T_{I}}\right)+(1-\alpha) \delta\left[\boldsymbol{v}-\boldsymbol{v}_{1}+2\left(\boldsymbol{v}_{1} \cdot \boldsymbol{n}\right) \boldsymbol{n}\right]\right\} .
$$

In Eqs. (2) and (3), $n_{I}$ is the equilibrium saturated vapor density at temperature $T_{I}, \sigma_{e}$ and $\sigma_{c}$ are the evaporation and condensation coefficient, respectively. The coefficient $\alpha$ assigns the probability that a molecule is either diffusively or specularly scattered after interacting with the liquid surface. A considerable amount of research effort has been put on validating the above model, developing new ones (Fujikawa et al., 2011; Frezzotti, 2011), and/or obtaining evaporation/condensation coefficients to be compared with experimental ones (Marek and Straub, 2001). Evaporation into near vacuum, occurring when the molecular flux from the vapor to the liquid can be neglected, has been studied by different methods to investigate the form of the distribution function of spontaneously evaporating molecules $f_{e}$. Most studies have been based on MD simulations of Lennard-Jones fluid (Zhakhovskii and Anisimov, 1997; Ishiyama et al., 2004a; Heinen, et al., 2016), as well as of molecular liquids (Ishiyama et al., 2004b). A kinetic model, described below in Section 4 , has been adopted in Frezzotti et al. (2005). The common conclusions can be summarized by saying that $f_{e}$ has the form of a half-range Maxwellian, as in Eq. (2), if the temperature of the liquid is well below the critical temperature $T_{c}$. In this condition, the estimated evaporation coefficients are close to unity, both for atomic and molecular liquids. A more or less pronounced temperature dependence of evaporation coefficients is found in the studies mentioned above, probably because of the different effective settings of the temperature attributed to the liquid in the interface region.

Models for kinetic boundary condition (KBC) have evolved to include the contribution of impinging molecular flux. In Ishiyama et al. (2013), Ishiyama and his coworkers have presented an improvement of a previous version of a model KBC, in which the form of the the distribution function of molecules emitted from the liquid surface, as the result of spontaneous evaporation and scattering of impinging molecules, has the form of a half-range Gaussian, whose anisotropy is related to the energy flux in a prescribed way. The model coefficients have been determined from MD simulations. MD simulations 
have also been used to develop models for condensation coefficients depending on the energy of vapor molecules impinging on the liquid surface, as described in Tsuruta et al. (1999) and Nagayama et al. (2015). The proposed analytical form of the condensation coefficient from the translational energy has been used in Bond and Struchtrup (2004) to interpret the temperature profiles experimentally observed during the evaporation of water (Fang and Ward, 1999).

\section{A summary of computational techniques for two-phase flows and their relationships with kinetic models}

It is convenient to describe and discuss both the existing and possible integrations of kinetic models with available two-phase computational techniques by considering two possible flow regimes.

In the first one, the curvature of the interface separating the liquid from the vapor region is much larger than some reference mean free path in the vapor. In this case, the Knudsen layer can be treated as locally one-dimensional, adhering to the interface. Furthermore if, as it often happens, the macroscopic characteristic flow time is much larger than the vapor mean free time, then the Knudsen layer behaves quasi-statically and the available results of the various studies mentioned in Section 1 can be used to match directly the hydrodynamic regions of the vapor and the liquid. This technique can be used in simple flow geometries, as show in Shusser et al. (2000), where the evaporation model in the explosive boiling of a liquid droplet was improved by adopting the jump relationships derived from approximate (Ytrehus, 1997) but accurate (Frezzotti, 2007) solutions of the Boltzmann equation. Evaporation/condensation sub-models have been also used in complex codes using Volume of Fluid (VoF) or Level Set Methods (LSM) to study more complex flow geometries. Evaporation/condensation rates based on a simple diffusive approximation are used in Schlottke and Weigand (2008) and Eisenschmidt et al. (2016), whereas linearized jump formulas, compatible with kinetic theory formulation are proposed in Hardt and Wondra (2008), in combination with a VoF scheme modified to allow mass, momentum and energy transfer between the vapor and liquid phases. In Strotos et al. (2008), various expressions for the evaporation mass flow rate were considered in the study of droplet depositing on a heated surface by VoF as well as by simplified droplet models. Unfortunately, the chosen kinetic model was based on Hertz-Knudsen formula which oversimplifies the kinetic layer structure by neglecting the effects of vapor advection and molecular back-scatter (Ytrehus, 1997).

When the typical size of liquid regions is of the order of the mean free path, kinetic regions might not only appear in the form of thin layers next to interfaces but extend to a significant fraction of the vapor flow field, which might also have a complicated geometry. Examples of this situations are evaporation/condensation phenomena in micro/nano droplets and bubbles systems or micro/nanofluidic devices (Lu et al., 2015). To simulate these flow regimes, there is no alternative to the direct coupling of the liquid phase, described by hydrodynamic equations (Navier-Stokes-Fourier) with the Boltzmann equation or a suitable kinetic model (Cercignani, 1988). On the vapor side, a boundary condition in the form given by Eq. (1) should be applied, whereas on the liquid side, the mass, momentum and energy fluxes should be prescribed by the overall balances involving molecules leaving from and impinging on interfaces. To the author's knowledge, examples of this kind of approach are scant and limited to simple one-dimensional geometries, where tracking the interfaces motion is not a difficult task. One dimensional evaporation and condensation flows between two liquid films have been studied in Onishi and Yamada (2003) by coupling a kinetic model for the vapor to the heat equations describing the temperature field in the liquid phase. The evaporation of a two-component spherical droplet has been studied in Sazhin et al. (2014) by coupling the continuum description in the liquid with the numerical solution of a system of Boltzmann equations for a binary mixture. A similar method has been used to compare MD simulations of the evaporation of a thin liquid film with a kinetic approach based on DSMC simulations of the vapor phase, combined with the heat transport equation in the liquid (Barbante et al., 2014).

A promising approach, capable of dealing with much more complex flow geometries, is described in Tiwari et al. (2013) where a mesh free scheme, called Finite Pointset Method, similar to the Smoothed Particles Hydrodynamics (SPH) method (Gingold and Monaghan, 1977), is used to construct a particle approximation of the Navier-Stokes equations describing the condensed phase. The Boltzmann equation, describing the vapor phase, is solved by the nowadays standard Direct Simulation Monte Carlo (DSMC) particle method (Bird, 1994). Unfortunately, the proposed version of the method did not allow mass transfer between the two phases, however modifications to include boundary conditions in the form of Eqs.(1-3) do not appear to pose prohibitive difficulties. Although examples are not yet present in the literature, it seems reasonable to anticipate that similar hybrid schemes, based on the coupling of VoF or LSM techniques with DSMC would also be feasible.

Although including kinetic models, the numerical techniques outlined above have limitations or drawbacks. Adopting 
jump formulas into VoF or LSM based two-phase codes has the advantage of using the same numerical scheme for both phases, provided that kinetic regions keep the shape of thin layers attached to interfaces. On the other hand, relaxing such assumption obliges combining distinct numerical techniques for the two phases into (very little developed) hybrid schemes. It is also worth observing that, in addition to the peculiar aspects of each numerical method, all the kinetic based schemes are limited by the assumption of dilute vapor phase, i.e. it is assumed that the average distance between molecules is much larger than the nominal molecular diameter. This is not the case in many relevant situations involving droplet evaporation (Sazhin, 2006) or explosive boiling (Shusser et al., 2000), where the vapor phase might be dense.

Although the kinetic theory of dense fluids is considerably less developed, approximate models are available to be used as a guidance. The ones described in the next Section have the capability of describing the fluid by a single kinetic equation. Interfaces are automatically formed between the condensed phase and the arbitrarily dense vapor, hence boundary conditions in the form of Eq. (1) are not necessary. However, the model can be used to formulate boundary conditions, as described in Frezzotti et al. (2005), Kobayashi et al. (2012) and Kon et al. (2014).

\section{Mean field kinetic approximation of a simple fluid}

An approximate (in the sense to be explained below) kinetic treatment of a simple fluid exhibiting phase separation, has been studied by several authors in De Sobrino (1967), Grmela (1971) and Karkheck and Stell (1981). A simple fluid is considered, composed by spherical and identical atoms of mass $m$. Atomic interactions are prescribed by a Sutherland potential in the form:

$$
\phi(r)=\left\{\begin{array}{cc}
+\infty & r<\sigma \\
-\phi_{\sigma}\left(\frac{r}{\sigma}\right)^{-\gamma} & r \geq \sigma
\end{array}\right.
$$

which results from the superposition of a hard sphere potential and an attractive soft potential tail. In Eq. (4), $r$ is the distance between the atomic centers, $\sigma$ is the hard sphere diameter whereas, $\phi_{\sigma}=\lim _{r \rightarrow \sigma^{+}} \phi(r)$ is the depth of the potential tail at $r=\sigma$, whereas $\gamma$ is a positive exponent tuning the effective range of the attractive forces. It should be noted that the algebraic form of the potential tail in Eq. (4) is not mandatory and adopting a more general form is possible. As discussed in Karkheck and Stell (1981), the following exact equation can be obtained for the fluid:

$$
\begin{aligned}
\frac{\partial f_{1}}{\partial t}+\boldsymbol{v} \cdot \nabla_{\boldsymbol{x}} f_{1}= & -\nabla_{\boldsymbol{v}} \cdot\left[\int_{\left\|\boldsymbol{x}_{1}-\boldsymbol{x}\right\|>\sigma} \hat{\boldsymbol{k}} \frac{d \phi}{d r} f_{2}\left(\boldsymbol{x}, \boldsymbol{v}, \boldsymbol{x}_{1}, \boldsymbol{v}_{1} \mid t\right) d \boldsymbol{x}_{1} d \boldsymbol{v}_{1}\right]+ \\
& \sigma^{2} \int\left[f_{2}\left(\boldsymbol{x}, \boldsymbol{v}^{*}, \boldsymbol{x}+\sigma \hat{\boldsymbol{k}}, \boldsymbol{v}_{1}^{*} \mid t\right)-f_{2}\left(\boldsymbol{x}, \boldsymbol{v}, \boldsymbol{x}-\sigma \hat{\boldsymbol{k}}, \boldsymbol{v}_{1} \mid t\right)\right] \times\left(\boldsymbol{v}_{r} \cdot \hat{\boldsymbol{k}}\right)^{+} d \boldsymbol{v}_{1} d^{2} \hat{\boldsymbol{k}} .
\end{aligned}
$$

Equation (5), quite generally, states that the rate of change of the 1-particle distribution function $f_{1}(\boldsymbol{x}, \boldsymbol{v} \mid t)$ is determined by the smooth force field generated by the tail potential (first term in the r.h.s. of Eq. (5)) and by the impulsive collisions whose effect is described by the second term in the r.h.s. of the same equation. Both terms contain the 2-particles distribution function $f_{2}\left(\boldsymbol{x}, \boldsymbol{v}, \boldsymbol{x}_{1}, \boldsymbol{v}_{1} \mid t\right)$ which, roughly speaking, describes the atoms distribution around a given one, occupying the position $\boldsymbol{x}$, with velocity $\boldsymbol{v}$ at time $t$. Therefore, Eq. (5) is exact but not closed. An approximate closure is achieved by following Enskog kinetic theory of the dense hard sphere gas (Resibois and DeLeener, 1977) and its more general formulation proposed by Ernst and Van Beijeren (1973). In this theory, the following assumption is made for $f_{2}$ in the hard sphere contribution:

$$
\begin{aligned}
& f_{2}\left(\boldsymbol{x}, \boldsymbol{v}, \boldsymbol{x}-\sigma \hat{\boldsymbol{k}}, \boldsymbol{v}_{1} \mid t\right)=\chi_{(h s)}(\boldsymbol{x}, \boldsymbol{x}-\sigma \hat{\boldsymbol{k}},\{n\}) f_{1}(\boldsymbol{x}, \boldsymbol{v} \mid t) f_{1}\left(\boldsymbol{x}-\sigma \hat{\boldsymbol{k}}, \boldsymbol{v}_{1} \mid t\right) \\
& f_{2}\left(\boldsymbol{x}, \boldsymbol{v}^{*}, \boldsymbol{x}+\sigma \hat{\boldsymbol{k}}, \boldsymbol{v}_{1}^{*} \mid t\right)=\chi_{(h s)}(\boldsymbol{x}, \boldsymbol{x}-\sigma \hat{\boldsymbol{k}},\{n\}) f_{1}\left(\boldsymbol{x}, \boldsymbol{v}^{*} \mid t\right) f_{1}\left(\boldsymbol{x}+\sigma \hat{\boldsymbol{k}}, \boldsymbol{v}_{1}^{*} \mid t\right)
\end{aligned}
$$

In Eq. (6), $\chi_{(h s)}(\boldsymbol{x}, \boldsymbol{x} \pm \sigma \hat{\boldsymbol{k}},\{n\})$ is the contact value of the pair correlation function (Resibois and DeLeener, 1977) in a hard sphere fluid in equilibrium with a density field $n(\boldsymbol{x} \mid t)$. In general, $\chi_{(h s)}$ is a functional of $n$, which can be obtained by the methods of statistical mechanics (Van Beijeren and Ernst, 1973). In many applications of the theory, the simpler original Enskog's formulation is used, in which the functional form of $\chi_{(h s)}$ is approximated by a function $\chi_{(E)}$ of the density, as follows:

$$
\chi_{(h s)}(\boldsymbol{x}, \boldsymbol{x} \pm \sigma \hat{\boldsymbol{k}}) \approx \chi_{E}\left[n\left(\boldsymbol{x} \pm \frac{\sigma}{2} \hat{\boldsymbol{k}} \mid t\right)\right],
$$

being $n\left(\boldsymbol{x} \pm \frac{\sigma}{2} \hat{\boldsymbol{k}} \mid t\right)$ the value of the fluid number density where two colliding hard sphere come to contact. The function $\chi_{E}(n)$ is easily related to the hard sphere fluid equation of state $p_{h s}=p_{h s}(n, T)($ Resibois and DeLeener, 1977):

$$
\chi_{E}(n)=\frac{1}{n b}\left(\frac{p^{h s}}{n \kappa T}-1\right), \quad b=\frac{2 \pi \sigma^{3}}{3} .
$$



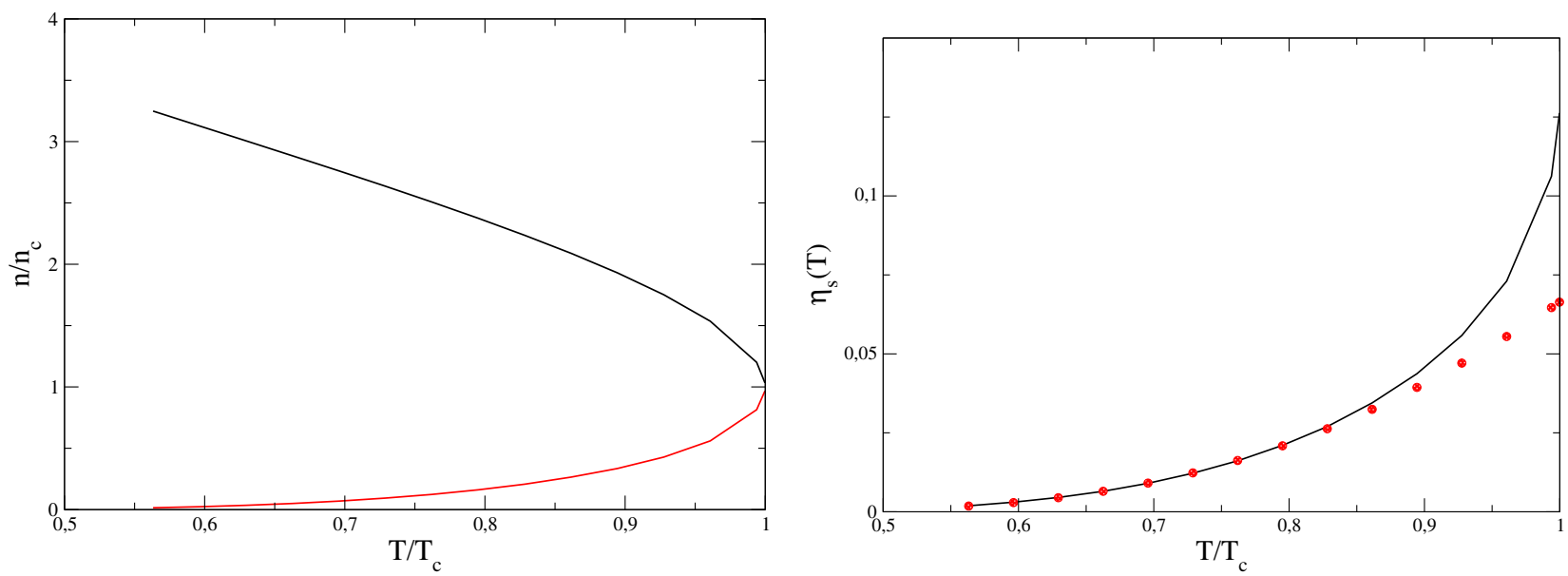

Fig. 1 Left- Liquid-vapor equilibrium coexistence curve from Eq. (14). Black solid line: liquid branch; Red solid line: vapor branch Right- Saturated vapor reduced density $\eta_{s}$ as a function of temperature and ClausiusClapeyron fit. Solid line: numerically computed $\eta_{s} ; \circ$ : Clausius-Clapeyron fit (Eq.(18)).

An even simpler closure for the tail contribution is obtained by ignoring correlations at distances larger than the hard sphere diameter $\sigma$ to write:

$$
f_{2}\left(\boldsymbol{x}, \boldsymbol{v}, \boldsymbol{x}_{1}, \boldsymbol{v}_{1} \mid t\right)=f_{1}(\boldsymbol{x}, \boldsymbol{v} \mid t) f_{1}\left(\boldsymbol{x}_{1}, \boldsymbol{v}_{1} \mid t\right), \quad r=\left\|\boldsymbol{x}_{1}-\boldsymbol{x}\right\|>\sigma, \quad \forall \boldsymbol{v}, \boldsymbol{v}_{1} .
$$

Inserting the relationships defined by Eqs. (6) and (9) into Eq. (5) leads to the following kinetic equation for the 1-particle distribution function:

$$
\frac{\partial f}{\partial t}+\boldsymbol{v} \cdot \nabla_{x} f+\frac{\boldsymbol{F}(\boldsymbol{x} \mid t)}{m} \cdot \nabla_{\boldsymbol{v}} f=C_{E}(f, f)
$$

where:

$$
\begin{aligned}
\boldsymbol{F}(\boldsymbol{x} \mid t)= & \int_{\left\|x_{1}-\boldsymbol{x}\right\|>\sigma} \frac{d \phi}{d r} \frac{\boldsymbol{x}_{1}-\boldsymbol{x}}{\left\|\boldsymbol{x}_{1}-\boldsymbol{x}\right\|} n\left(\boldsymbol{x}_{1} \mid t\right) d \boldsymbol{x}_{1}, \\
C_{E}(f, f)= & \sigma^{2} \int\left\{\chi_{(h s)}(\boldsymbol{x}, \boldsymbol{x}+\sigma \hat{\boldsymbol{k}},\{n\}) f\left(\boldsymbol{x}+\sigma \hat{\boldsymbol{k}}, \boldsymbol{v}_{1}^{*} \mid t\right) f\left(\boldsymbol{x}, \boldsymbol{v}^{*} \mid t\right)-\right. \\
& \left.\chi_{(h s)}(\boldsymbol{x}, \boldsymbol{x}-\sigma \hat{\boldsymbol{k}},\{n\}) f\left(\boldsymbol{x}-\sigma \hat{\boldsymbol{k}}, \boldsymbol{v}_{1} \mid t\right) f(\boldsymbol{x}, \boldsymbol{v} \mid t)\right\}\left(\boldsymbol{v}_{r} \cdot \hat{\boldsymbol{k}}\right)^{+} d \boldsymbol{v}_{1} d^{2} \hat{\boldsymbol{k}} .
\end{aligned}
$$

Equation (10), named Enskog-Vlasov equation (De Sobrino, 1967), describes a dense hard sphere fluid according to the standard or revised Enskog theory, subject to a self-consistent force field $\boldsymbol{F}$, generated by the potential soft tail. It is worth stressing that $\boldsymbol{F}$ is a linear functional of the number density $n(\boldsymbol{x} \mid t)$ which, in its turn, is obtained from $f(\boldsymbol{x}, \boldsymbol{v} \mid t)$ as:

$$
n(\boldsymbol{x} \mid t)=\int f(\boldsymbol{x}, \boldsymbol{v} \mid t) d^{3} \boldsymbol{v}
$$

It is not difficult to show that in a uniform fluid in equilibrium, described by Eq. (10), the equation of state has the following generalized Van der Waals form:

$$
p=p^{h s}(n, T)-\frac{2 \pi \sigma^{3}}{3} \frac{\gamma}{\gamma-3} \phi_{\sigma} n^{2},
$$

where $p^{h s}$ denotes the hard sphere contribution to the total pressure $p$. If the approximate (but accurate) expression for $p^{h s}$, proposed by Carnahan and Starling (1969), is used, then:

$$
p^{h s}(n, T)=n k T \frac{1+\eta+\eta^{2}-\eta^{3}}{(1-\eta)^{3}}, \quad \eta=\frac{\pi \sigma^{3} n}{6} .
$$

Using the above expression into Eq. (8) leads to the following approximation for $\chi_{E}(n)$ :

$$
\chi_{E}(n)=\frac{1}{2} \frac{2-\eta}{(1-\eta)^{3}}
$$



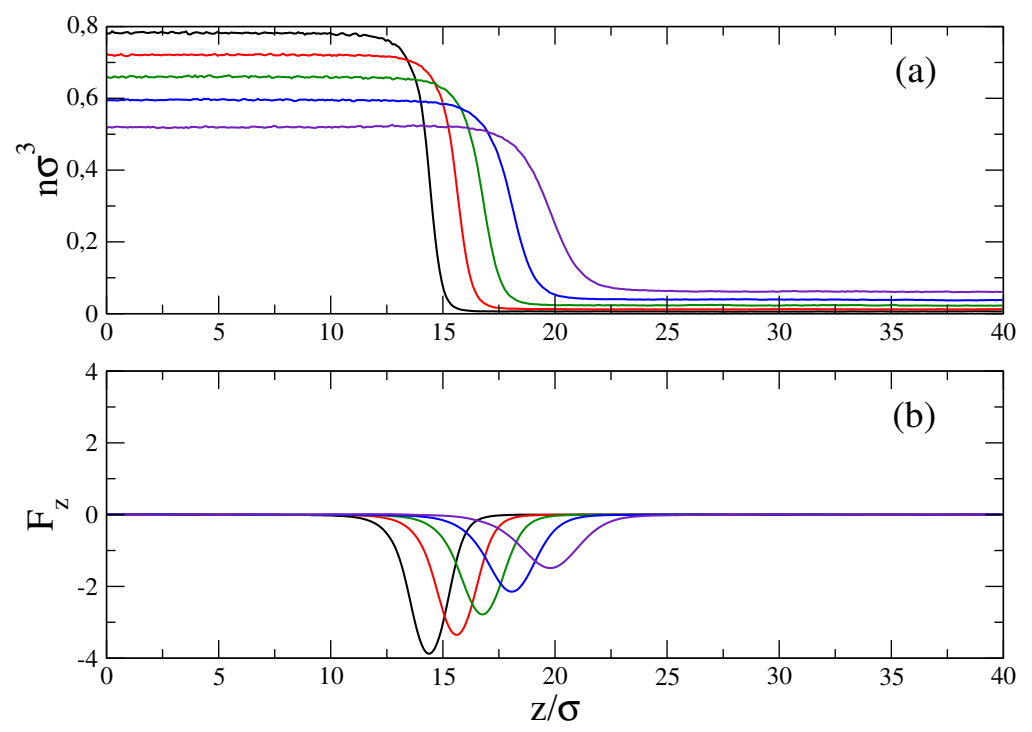

Fig. 2 (a) - Equilibrium density profiles computed from Eq. (19). Black solid line: $T / T_{c}=0.596$; Red solid line: $T / T_{c}=0.663$; Green solid line: $T / T_{c}=0.729$; Blue solid line: $T / T_{c}=0.795$; Indigo solid line: $T / T_{c}=0.862$. (b) - Self-consistent force field profiles, normalized to $k T_{c} / \sigma$; Solid lines colors correspond to temperature values as in panel (a).

Application of Maxwell's equal area rule to Eq. (14), in which the approximation (15) is used for $p^{h s}(n, T)$, leads to the following values for the fluid critical temperature $T_{c}$ and reduced density $\eta_{c}=\frac{\pi \sigma^{3}}{6} n_{c}, n_{c}$ being the critical density:

$$
\eta_{c}=0.130443, \quad T_{c}=0.094329 \frac{4 \gamma}{\gamma-3} \frac{\phi_{\sigma}}{k},
$$

and to the vapor liquid coexistence diagram depicted in Fig. 1. The saturated vapor density curve in Fig. 1 allows an easy estimation of the fluid latent heat of vaporization by using Clausius-Clapeyron equation. Limiting the analysis to the low density data, it is seen in Fig. 2 that the saturated vapor number density $n_{s}$ is well approximated by the ideal gas form of the Clausius-Clapeyron equation:

$$
n_{s}(T)=\frac{A}{R T} \exp \left(-\frac{\Delta H}{R T}\right),
$$

if $\Delta H \approx 5.3776 R T_{c}$ or $\Delta H \approx \frac{2 \gamma}{\gamma-3} \frac{\phi_{\sigma}}{k}$.

Equation (10) admits equilibrium solutions in which the temperature $T$ is uniform but the non-uniform density field $n(\boldsymbol{x})$ obeys the following equation when, for simplicity, it assumed that $n$ is made to depend only on a single space variable, say $z$ :

$$
k T \frac{d n}{d z}=n(z) F_{z}(z)+2 \pi \sigma^{2} n(z) k T \int_{-1}^{+1} k_{z} \chi_{E}\left[n\left(z-\frac{\sigma}{2} k_{z}\right)\right] n\left(z-\sigma k_{z}\right) d k_{z},
$$

where the $z$ component of the self-consistent field, $F_{z}$ is given by the following expression:

$$
F_{z}(z \mid t)=2 \pi \phi_{\sigma}\left[\sigma^{\gamma} \int_{\left|z-z_{1}\right|>\sigma} \frac{\left(z_{1}-z\right) n\left(z_{1} \mid t\right)}{\left|z-z_{1}\right|^{\gamma}} d z_{1}+\int_{\left|z-z_{1}\right| \leq \sigma}\left(z_{1}-z\right) n\left(z_{1} \mid t\right) d z_{1}\right] .
$$

Examples of numerical solutions of Eq. (19) in which a planar interface separates two semi-infinite liquid and vapor regions, in which the density takes values on the liquid-vapor coexistence curve of Fig. 1 far from the interface, are shown in Fig. 2, for different values of the temperature $T<T_{c}$. Figure 2 clearly shows the behavior and role of $F_{z}$ which is zero in the bulk of liquid and vapor regions but it takes a large value in the strongly inhomogeneous interface region, being directed from the vapor to the liquid in order to keep the condensed phase confined.

Before summarizing the specific applications of the Enskog-Vlasov model to non-equilibrium flows, it is worth mentioning that, as discussed in Karkheck and Stell (1981), the transport properties (shear and bulk viscosity, thermal conductivity) appearing in the hydrodynamic approximation of Eq. (10) are not affected by the potential tail, being determined only by the hard sphere collision term (12). Although this aspect represents a serious model drawback when it is desidered to match specific experimental flow conditions (Karkheck and Stell, 1981), it does not hinder the general capability of the Enskog-Vlasov equation to provide a sufficiently realistic description of the fluid behavior by tractable mathematical and numerical methods. 

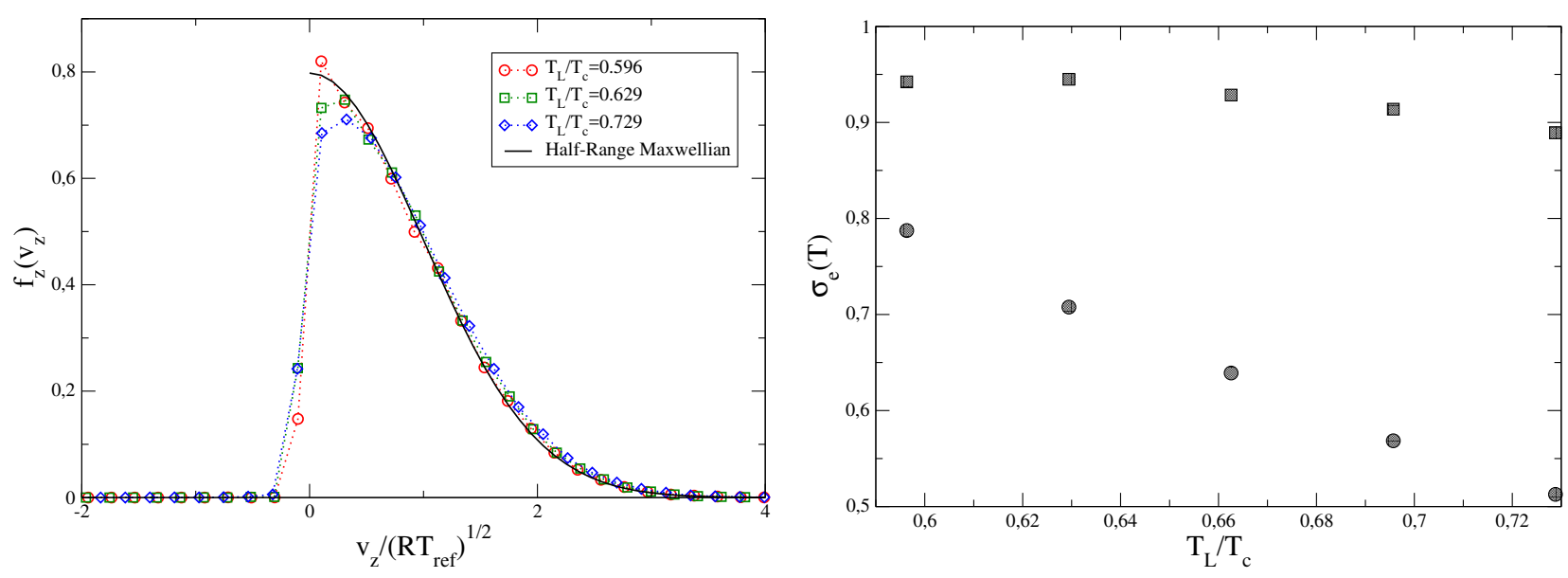

Fig. 3 Left- Reduced distribution function of molecular velocity component normal to the liquid-vapor interface, at the vapor-liquid interface. The black solid line represent the half-range Maxwellian. Symbols represent solutions of Eq. (10), for three values of the ratio $T_{L} / T_{c}$. Right- Temperature dependence of evaporation coefficient from Enskog-Vlasov simulations of the evaporation of a liquid film into near vacuum.

\subsection{Numerical aspects of Enskog-type kinetic equations}

Although describing a simple fluid, Enskog-Vlasov equation shares with all kinetic equations a relatively complex mathematical form which prevents obtaining non-trivial solutions without numerical methods. The computation and treatment of the force term in Eq. (10) does not pose any difficulty, irrespectively of the adopted numerical technique. The computation of the collision term, consisting in a 5-fold integral is more complex. Early attempts to solve the Enskog equation, in which $C_{E}$ keeps the form given in Eq. (12) but $\phi(r) \equiv 0$, were based on semi-regular numerical methods (Frezzotti and Sgarra, 1993). In these class of methods, developed for rarefied gas dynamics studies (Tcheremissine, 2006), the distribution function is discretized and the spatial advection term at the l.h.s. of Eq. (10) is approximated by any suitable finite difference scheme. The collision integral $C_{E}$ or its Boltzmann form are evaluated by Monte Carlo quadrature methods (Kalos and Whitlock, 1986). Later, less memory demanding particles schemes for the Enskog equation have been formulated in Montanero and Santos (1996), Montanero and Santos (1997) and Frezzotti (1997b), generalizing the well known DSMC particle scheme (Bird, 1994) to dense gases. As mentioned above, once the proper particle formulation for the Enskog contribution to Eq. (10) has been formulated, adding the self-consistent field term is a trivial task. The resulting scheme, described and discussed in detail in Barbante et al. (2015), requires a computational effort proportional to the number of simulation particles $N_{p}$ used to represent the distribution function $f$. It should be noted that the hard sphere diameter $\sigma$ is the natural spatial scale in Eq. (10) and the collision frequency dictated by the term $C_{E}$ is the natural time scale. Therefore, the computing time and memory request associated with the DSMC algorithm should be compared with molecular dynamics simulations which have comparable space and time scales. Results of not yet systematic comparisons show (Frezzotti, 1997b; Barbante et al., 2015) that the speed up of DSMC simulations with respect to MD, using the same particles number, depends on the dimensionality of the flow. In one-dimensional flows DSMC runs about two orders of magnitude faster than MD; in two-dimensional simulations the speed up reduces to one order of magnitude. It is estimated that DSMC and MD would require similar computing times in three-dimensional flows. However, estimates are based on relatively unsophisticated versions of the algorithm. The reason of the DSMC speed up reduction lies in the growing effort required to compute the self-consistent field. The latter is proportional to the product of the number of spatial cells $N_{c}$ times the number $\tilde{N}_{c}$ of spatial cells within the cutoff radius of $\phi(r)$. Considerable reductions of the computing time can then be obtained by adopting adaptive grids in place of simple uniform grids. Furthermore, standard parallelization strategies, based on domain decomposition can be easily and efficiently implemented. It is also worth mentioning that a deterministic fast spectral method has been recently proposed (Wu et al., 2015) to obtain accurate solutions of the Enskog equation.

\subsection{An alternative kinetic scheme:CBA}

For the sake of completeness, the Consistent Boltzmann Algorithm (CBA) should be considered as a kinetic scheme capable of simulating dense fluids and liquid-vapor systems. As its name suggests, in its initial formulation by Alexander, Garcia and Alder (1997), the method was proposed as a direct modification of DSMC, not as a numerical scheme to solve an underlying kinetic equation. The authors observed that, in the original DSMC scheme for an ideal gas the spatial 
distribution of simulations particles within a cell is not used in the algorithm. However, if collision partners are given prescribed opposite spatial displacements, after the velocity changes following a binary collision, then the gas becomes non-ideal. More precisely, if the local collision rate is enhanced by a factor $\chi_{E}(n)$, as in the Enskog equation, and the mentioned displacement step is added to the usual DSMC scheme, then the fluid equation of state deviates from the ideal behavior, since the application of post-collision spatial displacements is the CBA counterpart of the non-local interaction in the Enskog equation. Properly tuning the displacement extension allow obtaining an arbitrary equation of state and, in particular van der Waals' (Garcia et al., 1997). CBA particle scheme has also been used to study vapor liquid systems and the formation of droplets in the van der Waals fluid (Hadjiconstantinou et al., 2000). It should also be noted that, although initially developed directly as an extension of the DSMC scheme, the underlying Enskog-like kinetic equation, whose particle scheme realization corresponds to CBA has been described in Garcia and Wagner (2000). Although CBA has not been been used for a systematic study of evaporation/condensation flows, its computational structure seems to be simpler than the DSMC implementation of the Enskog-Vlasov equation.

\section{Applications of Enskog-Vlasov model to liquid vapor systems}

In this section a few studies aiming at assessing the capabilities of Enskog-Vlasov model to vapor-liquid systems out of equilibrium are summarized. The first example is provided in Frezzotti et al. (2005) where the evaporation of a thin liquid film is studied, assuming that the vapor density is so low that collisions in the vapor phase can be neglected. In this case, the distribution function in the gas phase coincides with $f_{e}$, according to Eq. (1). Therefore, any method capable of computing the liquid-vapor system in such approximation can obtain the form of the distribution function of molecules spontaneously emitted by the liquid bulk. Moreover, comparison of the computed mass flux $J_{m}$ with the one given by Eq. (2) allows for the calculation/estimation of the evaporation coefficient $\sigma_{e}$. As mentioned above, the method has been applied to study mono and polyatomic fluids, in combination with molecular dynamics simulations in Zhakhovskii and Anisimov (1997), Ishiyama et al. (2004a), Ishiyama et al. (2004b) and Heinen et al. (2016). The studies conclude that the form of $f_{e}$ agrees with the half range Maxwellian of Eq. (2) when the liquid temperature $T_{L}$ is well below the critical temperature, as shown in the left panel of Fig. 3. Also, evaporation coefficients close to unity have been found at low liquid temperature. Remarkably, application of the Enskog-Vlasov model to the same problem produced very similar results about the shape of $f_{e}$, which was found to be very close to a half-range Maxwellian far from $T_{c}$, i.e. when the vapor is nearly ideal and the model expressed by Eq. (1) can be applied. The evaporation coefficient, computed from Enskog-Vlasov model, is show in the right panel of Fig. 3 as function of $T_{L} / T_{c}$. Squares mark values obtained by taking into account the interface cooling, where circles mark values obtained by making reference to the saturated vapor pressure and the thermostatted liquid temperature $T_{L}$.

Studies aimed at using Enskog-Vlasov model to derive KBC have been described in Kobayashi et al. (2012) and Kon et al. (2014). In the latter paper, one dimensional simulations of the steady evaporation/condensation flow between two planar liquid films kept at different temperatures are performed in conditions where the vapor is nearly ideal. On the ground of the accurate numerical results, the authors propose that the overall distribution function of molecules emerging from the liquid surface is represented by a half-range Maxwellian. However, the contribution of "reflected" molecules, which superposes to spontaneous evaporation, is different for evaporation and condensation. In the first case, the expression is consistent with a diffuse reflection with an evaporation coefficient close to unity $\left(\sigma_{e}=0.87\right)$, whereas in the second case, the condensation coefficient $\sigma_{c}$ is not constant but depends on the impinging molecular flux. As in the case of the model KBC mentioned in Section 2, it is to be observed that additional work is needed to reconcile the various proposed formulations and to extend the models validation to more complex flows and experiments.

As mentioned in Section 4.1, the particle scheme for the numerical solution of Eq. (10) generally requires less computational effort than a similar MD simulation. This characteristic can be exploited to use a larger particle simulation number, in order to reduce statistical noise in the estimation of macroscopic fields, particularly in unsteady flow configurations where time averaging can be applied to short intervals. It is also worth observing that Eq. (10) should be provided with boundary conditions to describe fluid-wall interaction. However, the formulation of gas-wall boundary conditions used in non-equilibrium dilute gas flows (Cercignani, 1988) would not provide a realistic description of liquid-solid interaction. Hence, an approximate model has been formulated in the same spirit of the Enskog-Vlasov equation (Barbante et al., 2015). More precisely, the model assumes that the molecular interaction between fluid and wall molecules has the form of Eq. (4), although with different hard sphere diameter and soft tail, in general. Again, the effects of the hard sphere potential and soft potential tail are respectively treated by a collision integral, in the form of Eq. (12) and a force field to 

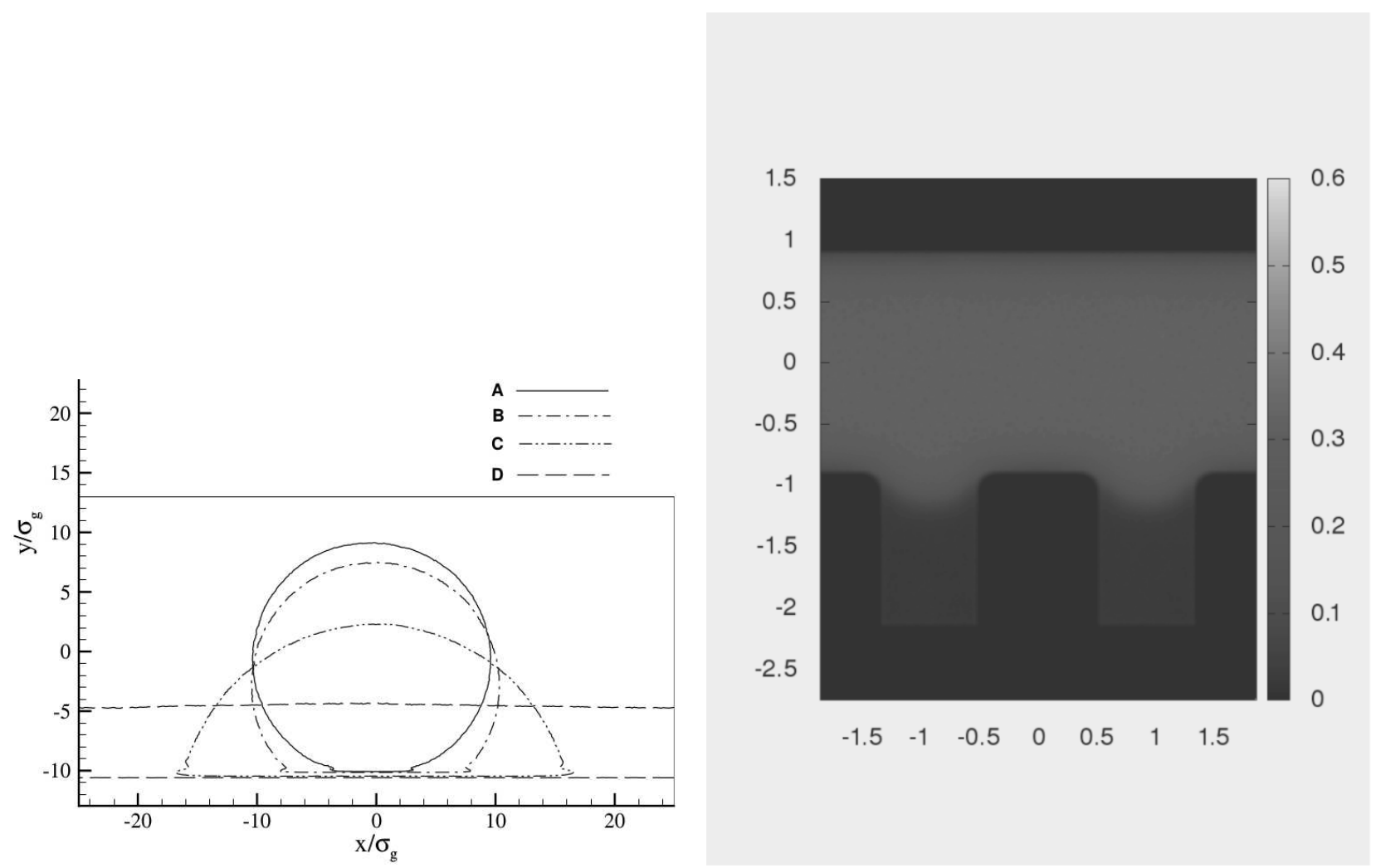

Fig. 4 Left- Shape of an isolated drop in contact with a solid wall for different fluid-wall interaction strength. From case $A$ to $D$ wall becomes increasingly hydrophilic (Barbante et al., 2015). Right- Grey scale density map of a liquid film in contact with a grooved wall. $x$ and $y$ axis normalized to domain width $L_{x}$ and heigth $L_{y}$, respectively. $L_{x}=L_{y}=30 \sigma$.

be added to the fluid-fluid interaction terms already present in Eq. (10). The advantage of the fluid-wall model is that the resulting kinetic equation can be solved without changing the numerical scheme. Moreover, the model parameters allow to tune the liquid-solid contact angle by changing the strength of the interaction. Examples of applications of numerical solutions of Eq. (10) are presented in Figs. 4 and 5. The equilibrium shape of an isolated drop in contact with a solid wall is shown on the left panel of Fig. 4. The four curves, $A-D$, have been obtained changing the fluid-wall parameters to produce increasingly hydrophilic walls. The right panel of Fig. 4 shows the grey scale map of the fluid density in a film deposited onto a grooved wall. The formation of menisci and vapor regions between the wall "teeth" are clearly visible. In Barbante et al. (2015), the stability of liquid bridges joining solid walls has been investigated studying spatially twodimensional and unsteady version of Eq. (10). Figure 5 shows two fluid density snapshots obtained immediately before and after the liquid bridge breaking.

\section{Conclusions}

As declared at the start of this paper, a review has been presented of the studies of evaporation/condensation flows from the point of view of the kinetic theory of fluids. The literature described in Section 2, devoted to the classical studies of the Knudsen layer structure in a dilute vapor, shows that the general gas dynamic properties have been made clear and that the extensions to polyatomic gases and mixtures have somehow completed the necessary framework to analyze experiments and applications. However, the little studied case in which the evaporating/condensing vapor is composed by a number of polyatomic species (Shishkova et al., 2013) has to be further investigated, in view of its relevance for applications. A complete kinetic theory study of the Knudsen layer structure cannot be obtained without reliable models of kinetic boundary conditions to couple the vapor to the liquid phase. In this respect, available investigations, mostly based on Molecular Dynamics simulations, point at a revision of the "standard" model. Suggested changes take the form of energy dependent condensation coefficients or anisotropic shape of the distribution function of molecules emitted from the liquid phase. It is clear that additional efforts are to be made to obtain a unified view of KBC. Moreover, the various proposed models should be validated through realistic flow simulations to be possibly compared with experiments. The 

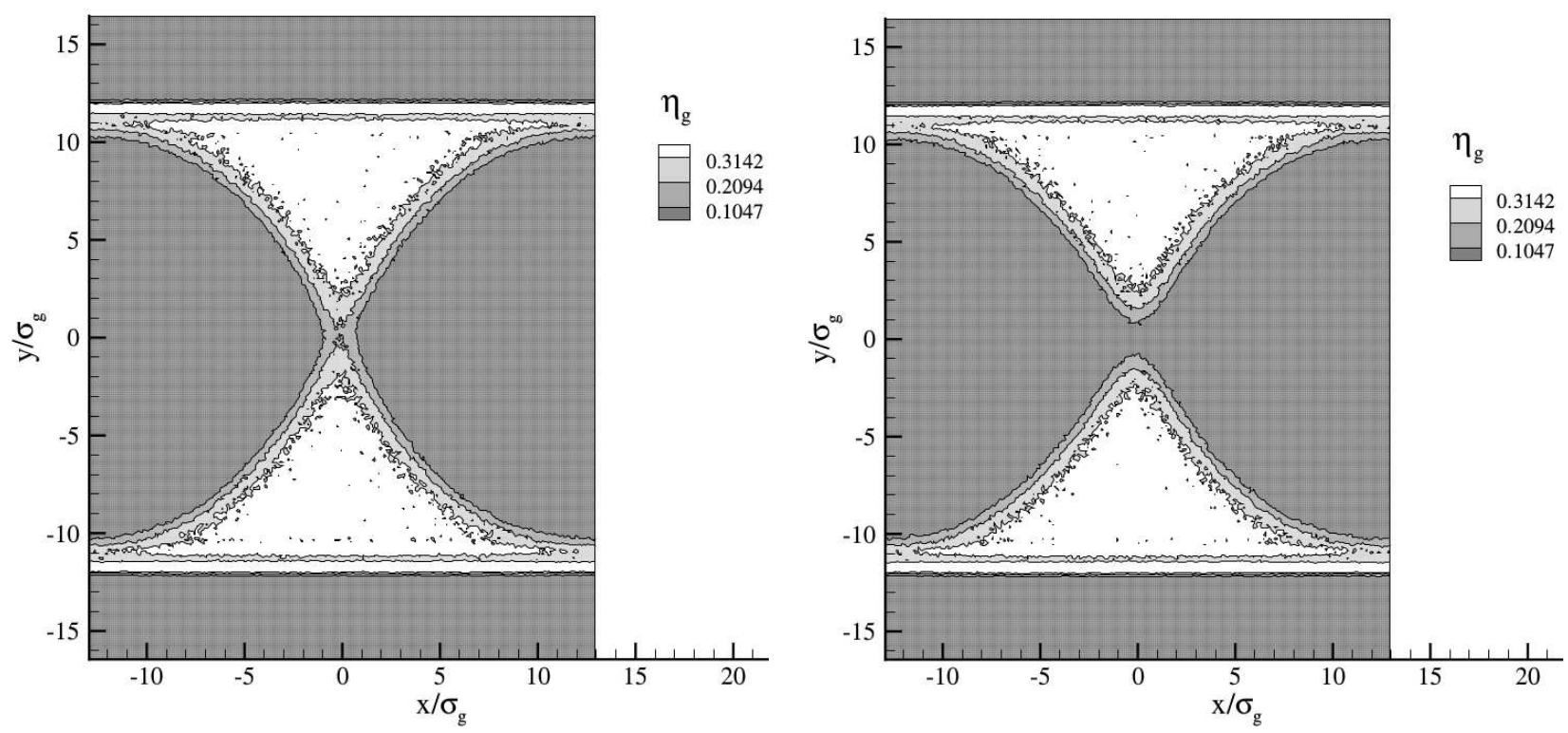

Fig. 5 Enskog-Vlasov simulation of the breaking of a liquid bridge between solid planar walls (Barbante et al., 2015)

latter aspect is also to be further developed. As a matter of fact, the use of consistent kinetic models in the simulation of liquid-vapor systems is still limited to very simple flow geometries. However, the adoption of kinetic jump formulas to include evaporation/condensation in two-phase flows simulation codes or the development of hybrid hydrodynamickinetic schemes would allow assessing the overall sensitivity of the flow properties with respect to the adopted KBC model, as well extending the modeling capabilities of multiphase micro/nano flows. As observed above, the kinetic models described in Section 4 have a number of advantages. They provide a mathematical model to investigate nonequilibrium and non-ideal vapor flows; the associated numerical schemes require smaller computational effort, when compared with MD simulations; their estimations of evaporation/condensation coefficients and vapor-liquid scattering properties of simple fluids exhibit good agreement with MD results. Model extensions to mixtures and polyatomic species are possible, however they should be considered with caution in order to avoid excessively artificial formulations. The role of Enskog-Vlasov model is not to compete with MD techniques but to help understanding the complexities of interfacial phenomena. 


\section{References}

Alexander F.J., Garcia, A.L., Alder, B.J., The consistent Boltzmann algorithm for the van der Waals equation of state, Physica A, Vol.240, No.1-2 (1997), pp. 196-201.

Anderson, D. M., McFadden, G. B. and Wheeler, A. A., Diffuse interface methods in fluid mechanics, Annual Review of Fluid Mechanics, Vol.30, No.1 (1998), pp. 139-165.

Aoki, K., Takata, S. and Kosuge, S., Vapor flows caused by evaporation and condensation on two parallel plane surfaces: Effect of the presence of a noncondensable gas, Physics of Fluids, Vol.10, No.6 (1998), pp. 1519-1533.

Barbante, P., Frezzotti, A. and Gibelli, L., A Comparison of Molecular Dynamics and Diffuse Interface Model Predictions of Lennard-Jones Fluid Evaporation, Proceedings of the 29th International Symposium on Rarefied Gas Dynamics (2014), pp. 893-900.

Barbante, P., Frezzotti, A., Gibelli, L., A kinetic theory description of liquid menisci at the microscale, Kinetic and Related Models, Vol.8, No.2 (2015), pp. 235-254.

Bird, G.A., Molecular Gas Dynamics and the Direct Simulation of Gas Flows (1994), Clarendon Press.

Bond, M. and Struchtrup, H., Mean evaporation and condensation coefficients based on energy dependent condensation probability, Physical Review E, Vol.70, No.6 (2004), 061605.

Carnahan, N.F. and Starling, K.E., Equation of state for non-actracting rigid spheres, Journal of Chemical Physics, Vol. 51, No.2 (1969), pp. 635-636.

Cercignani, C., Strong evaporation of a polyatomic gas, Proceedings of 12th International Symposium on Rarefied Gas Dynamics, Part 1 (1980), pp. 305-320.

Cercignani, C., The Boltzmann Equation and its Applications (1988), Springer-Verlag.

Davis, H.T., Kinetic theory of strongly inhomogeneous fluids, University of Minnesota Supercomputer Institute Research Report UMSI 92/78 (1992).

De Sobrino, L., On the kinetic theory of a Van der Waals gas, Canadian Journal of Physics, Vol. 45, No.2 (1967), pp. $363-385$

Eisenschmidt, K., et al., Direct numerical simulations for multiphase flows: An overview of the multiphase code FS3D, Applied Mathematics and Computation, Vol.272, No.2 (2016), pp. 508-517.

Fang, G., Ward, C.A., Temperature measured close to the interface of an evaporating liquid, Physical Review E, Vol.59, No.1 (1999), pp. 417-428.

Frezzotti A., Sgarra C., Numerical analysis of a shock-wave solution of the Enskog equation obtained via a Monte Carlo method, Journal of Statistical Physics, Vol.73, No.1-2 (1993), pp. 193-207.

Frezzotti, A., A numerical investigation of the steady evaporation of a polyatomic gas, European Journal of MechanicsB/Fluids, Vol. 26, No.1 (2007), pp. 93-104.

Frezzotti, A., Boundary conditions at the vapor-liquid interface, Physics of Fluids, Vol.23, No.3 (2011), pp. 030609.

Frezzotti, A., Ghiroldi, G.P., Gibelli, L. and Bonucci, A., DSMC simulation of rarefied gas mixtures flows driven by arrays of absorbing plates, Vacuum, Vol.103, No.5 (2014), pp. 57-67.

Frezzotti, A., Gibelli, L. and Lorenzani, S., Mean field kinetic theory description of evaporation of a fluid into vacuum, Physics of Fluids, Vol.17, No.1 (2005), 012102.

Frezzotti, A., Kinetic theory description of the evaporation of multi-component substances, Proceedings of the 20th International Symposium on Rarefied Gas Dynamics (1997a), pp. 837-846.

Frezzotti,A., A particle scheme for the numerical solution of the Enskog equation, Physics of Fluids, Vol.9, No.5 (1997b), pp.1329-1335.

Frezzotti, A., Numerical study of the strong evaporation of a binary mixture, Fluid Dynamics Research, Vol.8, No.5-6 (1991), pp. 175-187.

Frezzotti, A., and Ytrehus, T., Kinetic theory study of steady condensation of a polyatomic gas, Physics of Fluids, Vol.18. No.2 (2006), pp. 027101.

Fujikawa, S., Yano, T. and Watanabe, M., Vapor-liquid interfaces, bubbles and droplets (2011), Springer.

Garcia, A.L. and Wagner, W., The Limiting Kinetic Equation of the Consistent Boltzmann Algorithm for Dense Gases, Journal of Statistical Physics Vol.101, No. 5-6 (2000), pp. 1065-1086.

Garcia, A.L., Alexander, F.J. and Alder, B. J., A Particle method with adjustable transport properties - the generalized consistent Boltzmann algorithm, Journal of Statistical Physics, Vol.89, No.1-2 (1997), pp. 403-409.

Gingold, R.A. and Monaghan, J.J., Smoothed particle hydrodynamics: theory and application to non-spherical stars. 
Monthly Notice of the Royal Astronomical Society, Vol. 181, No.3 (1977), pp. 375-389.

Grmela, M., Kinetic equation approach to phase transitions, Journal of Statistical Physics, Vol.3, No.3 (1971), pp. 347364.

Hadjiconstantinou, N.G., Garcia, A.L., Alder, B.J., The surface properties of a van der Waals fluid, Physica A, Vol.281, No.1-4 (2000), pp. 337-347.

Hardt, S. and Wondra, F., Evaporation model for interfacial flows based on a continuum-field representation of the source terms, Journal of Computational Physics Vol. 227, No.11 (2008), pp. 5871-5895.

He, X. and Doolen, G.D., Thermodynamic foundations of kinetic theory and lattice Boltzmann models for multiphase flows, Journal of Statistical Physics, Vol. 107, No.1 (2002), pp. 309-328.

Heinen, M., Vrabec, J. and Fischer, J., Evaporation: Influence of heat transport in the liquid on the interface temperature and the particle flux, Journal Of Chemical Physics, Vol. 145, No.8 (2016), pp. 081101.

Hertz, H., On the evaporation of liquids, especially of mercury, in empty space, Annalen der Physik, Vol.253, No.10 (1882), pp. 177-193. (in German).

Ishiyama, T., Fujikawa, S., Kurz, T. and Lauterborn W., Nonequilibrium kinetic boundary condition at the vapor-liquid interface of argon, Physical Review E, Vol.88, No.4 (2013), pp. 042406.

Ishiyama, T., Yano, T. and Fujikawa, S., Molecular dynamics study of kinetic boundary condition at an interface between argon vapor and its condensed phase, Physics of Fluids, Vol.16, No.8 (2004a), pp. 2899-2906.

Ishiyama, T., Yano, T. and Fujikawa, S., Molecular dynamics study of kinetic boundary condition at an interface between a polyatomic vapor and its condensed phase, Physics of Fluids, Vol.16, No.12 (2004b), pp. 4713-4726.

Kalos, M.H., Whitlock, P.A., Monte Carlo Methods (1986), Wiley.

Karkheck, J. and Stell, G., Mean Field Kinetic Theories, Journal of Chemical Physics, Vol. 75, No.3 (1981), pp. 14751487.

Knudsen, M., The maximum evaporation rate of mercury, Annalen der Physik, Vol.352, No.13 (1915), pp. 697-708. (in German).

Kobayashi, K., Ohashi, K. Watanabe, M., Numerical analysis of vapor-liquid two-phase system based on the EnskogVlasov equation, AIP Conference Proceedings, Vol. 1501 (2012), pp. 1145-1153.

Kon, M., Kobayashi, K. and Watanabe M., Method of determining kinetic boundary conditions in net evaporation/condensation, Physics of Fluids, Vol.26, No.7 (2014), pp. 072003.

Kosuge, S., Aoki, K. and Hatano, M., Slow evaporation and condensation on a spherical droplet in the presence of a noncondensable gas, Physics of Fluids, Vol.22, No.6 (2010), pp. 067101.

Lu, Z., Narayanan S., Wang. E., Modeling of evaporation from nanopores with nonequilibrium and nonlocal effects, Langmuir, Vol.31, No.36 (2015), pp. 9817-9824.

Marek, M. and Straub, J., Analysis of the evaporation coefficient and the condensation coefficient of water, International Journal of Heat and Mass Transfer, Vol. 44, No.1 (2001), pp. 39-53.

Montanero, J.M. and Santos, A., Monte Carlo simulation method for the Enskog equation, Physical Review E, Vol.54, No.1 (1996), pp. 438-444.

Montanero, J.M. and Santos, A., Simulation of the Enskog equation á la Bird, Physics of Fluids, Vol.9, No.7 (1997), pp. 2057-2060.

Nagayama, G., Takematsu, M., Mizuguchi, H. and Tsuruta, T., Molecular dynamics study on condensation/evaporation coefficients of chain molecules at liquidvapor interface, Journal of Chemical Physics Vol. 143, No.1 (2015), 014706.

Ohse, R.W., Babelot, J.F., Frezzotti, A., Long, K.A. and Magill, J., Equation of state of Uranium Oxide: Mach-disk investigation of transient laser-induced vaporization of UO2 up to $5000 \mathrm{~K}$, High Temperatures - High Pressures, Vol.12, No.5 (1980), pp. 537-548.

Onishi, Y. and Yamada, K., Evaporation and condensation from or onto condensed phase with an internal structure, Proceedings of the 23th International Symposium on Rarefied Gas Dynamics (2003), pp. 630-637.

Persad, A.H. and Ward C.A., Expressions for the evaporation and condensation coefficients in the Hertz-Knudsen relation, Chemical Reviews, Vol.116, No.14 (2016), pp. 7727-7767.

Piechór, K., Non-local Korteweg stresses from kinetic theory point of view, Archive of Mechanics, Vol.60, No.1 (2008), pp. 23-58.

Resibois, P. and DeLeener, M., Classical kinetic theory of fluids (1977), J. Wiley \& Sons.

Sazhin, S. S., Advanced models of fuel droplet heating and evaporation, Progress in Energy and Combustion Science, Vol.32, No.2 (2006), pp. 162-214. 
Sazhin, S.S., Shishkova, I.N., Al Qubeissi, M., Heating and evaporation of a two-component droplet: Hydrodynamic and kinetic models, International Journal of Heat and Mass Transfer, Vol. 79, No.1 (2014), pp. 704-712.

Schlottke J. and Weigand B., Direct numerical simulation of evaporating droplets, Journal of Computational Physics, Vol. 227, No.10 (2008), pp. 5215-5237.

Schrage, R.W., A Theoretical Study of Interphase Mass Transfer (1988), Columbia University Press.

Shishkova I.N., Sazhin S.S. and Xie, J.F., A solution of the Boltzmann equation in the presence of inelastic collisions, Journal of Computational Physics, Vol. 232, No.1 (2013), pp. 87-99.

Shusser, M., Ytrehus, T. and Weihs, D., Kinetic theory analysis of explosive boiling of a liquid droplet, Fluid Dynamics Research, Vol.27, No.6 (2000) pp. 353-365.

Sibold, D., Urbassek, M., Monte Carlo study of Knudsen layers in evaporation from elemental and binary media, Physics of Fluids A, Vol.5, No.1 (1993), pp. 243-255.

Soga, T., A kinetic theory analysis of evaporation and condensation of a diatomic gas, Physics of Fluids, Vol.28, No.5 (1985), pp. 1280-1285.

Sone, Y., Kinetic theoretical studies of the half-space problem of evaporation and condensation, Transport Theory and Statistical Physics, Vol.29, No.2-5 (2000), pp. 227-260.

Strotos, G., Gavaises, M., Theodorakakos, A. and Bergeles, G., Numerical investigation on the evaporation of droplets depositing on heated surfaces at low Weber numbers, International Journal of Heat and Mass Transfer, Vol.51, No.7-8 (2008), pp. 1516-1529.

Takata, S. and Golse, F., Half-space problem of the nonlinear Boltzmann equation for weak evaporation and condensation of a binary mixture of vapors, European Journal of Mechanics, B/Fluids, Vol.26, No.1 (2007), pp. 105-131.

Tcheremissine, F.G., Solution to the Boltzmann kinetic equation for high-speed flows, Computational Mathematics and Mathematical Physics, Vol.46, No.2 (2006), pp. 315-329.

Tiwari, A. and Abraham, J., Dissipative particle dynamics simulations of liquid nanojet breakup, Microfluidics and Nanofluidics, Vol. 4, No.3 (2008), pp. 227-235.

Tiwari, S., Klar, A., Hardt, S. and Donkov, A., Coupled solution of the Boltzmann and Navier-Stokes equations in gasliquid two phase flow, Computers and Fluids, Vol.71, No.1 (2013), pp. 283-296.

Tsuruta, T., Tanaka, H. and Masuoka, T., Condensation/evaporation coefficient and velocity distributions at liquid-vapor interface, International Journal of Heat and Mass Transfer, Vol.42, No.22 (1999), pp. 4107-4116.

Van Beijeren, H. and Ernst, M. H., The modified Enskog equation, Physica, Vol. 68, No.3 (1973), pp. $437-456$.

Wu, L., Zhang, Y. and Reese, J.M, Fast spectral solution of the generalized Enskog equation for dense gases, Journal of Computational Physics, Vol. 303, No.1 (2015), pp. 66-79.

Ytrehus, T., Molecular flow effects in evaporation and condensation at interfaces, Multiphase Science and Technology, Vol.9, No.3 (1997), pp. 205-327.

Zhakhovskii, V.V. and Anisimov, S.I., Molecular dynamics simulation of evaporation of a liquid, Journal of Experimental and Theoretical Physics, Vol.84, No.4 (1997) pp. 734-745. 